\title{
Value of breast MRI omics features and clinical characteristics in Breast Imaging Reporting and Data System (BI-RADS) category 4 breast lesions: an analysis of radiomics-based diagnosis
}

\author{
Qian Cui ${ }^{1}$, Liang Sun ${ }^{2}$, Yu Zhang ${ }^{3}$, Zimu Zhao ${ }^{1}$, Shuo Li $^{1}$, Yajie Liu ${ }^{1}$, Hongwei Ge ${ }^{2}$, Dongxue Qin ${ }^{1 \#}$, \\ Yiping Zhao ${ }^{1 \#}$ \\ ${ }^{1}$ Department of Radiology, Second Affiliated Hospital of Dalian Medical University, Dalian, China; ${ }^{2}$ College of Computer Science and Technology, \\ Dalian University of Technology, Dalian, China; ${ }^{3}$ Department of Medical Imaging, Cancer Hospital of China Medical University, Liaoning Cancer \\ Hospital \& Institute, Shenyang, China \\ Contributions: (I) Conception and design: Y Zhao, Q Cui; (II) Administrative support: D Qin; (III) Provision of study materials or patients: Z Zhao, \\ S Li, Y Liu; (IV) Collection and assembly of data: L Sun, Y Zhang, H Ge; (V) Data analysis and interpretation: Q Cui; (VI) Manuscript writing: All \\ authors; (VII) Final approval of manuscript: All authors. \\ "These authors contributed equally to this work. \\ Correspondence to: Dongxue Qin; Yiping Zhao. Department of Radiology, Second Affiliated Hospital of Dalian Medical University, Dalian 116027, \\ China. Email: 17709875038@163.com; yipingzhao1975@dmu.edu.cn.
}

Background: The Breast Imaging Reporting and Data System (BI-RADS) category 4 breast lesions is categorized into $4 \mathrm{~A}, 4 \mathrm{~B}$, and $4 \mathrm{C}$, which reflect an increasing malignancy potential from low $(2-10 \%)$ moderate (10-50\%) and high (50-95\%). Determining the benign and malignant of BI-RADS category 4 breast lesions is very important for accurate diagnosis and follow-up treatment. This study aimed to explore the value of breast magnetic resonance imaging (MRI) omics features and clinical characteristics in the assessment of BI-RADS category 4 breast lesions.

Methods: This retrospective study analyzed 96 lesions (39 benign and 57 malignant) from 92 patients diagnosed with MRI BI-RADS category 4 lesions in the Second Affiliated Hospital of Dalian Medical University between May 2017 and December 2019. The lesions were sub-categorized as BI-RADS 4A, 4B, or 4C based on the MRI findings. An imaging omics analysis model was applied to extract the MRI features. The positive predictive value (PPV) of each subcategory was calculated, and the area under the curve (AUC) was used to describe the efficiency for different diagnoses. Moreover, we analyzed 17 clinical indicators to assess their diagnostic value for BI-RADS category 4 breast lesions.

Results: The PPVs of BI-RADS 4A, 4B, and 4C were 7.1\% (2/28), 41.2\% (7/17), and 94.1\% (48/51), respectively. The AUC, sensitivity, and specificity were $0.919,84.2 \%$, and $92.3 \%$, respectively. The combination of $\mathrm{T}_{1}$-weighted images $\left(\mathrm{T}_{1} \mathrm{WI}\right)$ with dynamic contrast-enhanced (DCE) MRI yielded the best diagnostic results among all dual sequences. Two clinical indicators [progesterone receptor (PR) and Ki67 expression] achieved an AUC almost equal to 1.0. The radiomics and redundancy reduction methods reduced the clinical data features from 1,233 to 14 .

Conclusions: High diagnostic performance can be achieved in distinguishing malignant breast BIRADS category 4 lesions using the combination of $\mathrm{T}_{1} \mathrm{WI}$ and DCE in MRI. Combining the PR and Ki-67 expression variables can further improve MRI accuracy for breast BI-RADS category 4 lesions.

Keywords: Breast neoplasms; magnetic resonance imaging (MRI); radiomics pathology; clinical indicators

Submitted Sep 18, 2021. Accepted for publication Nov 04, 2021.

doi: 10.21037/atm-21-5441

View this article at: https://dx.doi.org/10.21037/atm-21-5441 


\section{Introduction}

Breast cancer is one of the leading causes of cancer-related death among women, both in China and worldwide $(1,2)$. Early diagnosis of breast cancer can significantly prolong the survival of patients and reduce the cost of treatment $(3,4)$. The Breast Imaging Reporting and Data System (BIRADS) plays an essential role in diagnosing breast diseases. BI-RADS is a classification system proposed by the American College of Radiology (ACR) in 1986 with the original report released in 1993. The latest edition is BI-RADS 5 [2013] (5). According to mammography findings, breast lesions can be sorted into categories numbered 0 through 6 by the BIRADS (where a larger number reflects greater malignancy), with category 4 referring to suspicious abnormality (6). The BI-RADS category 4 is further categorized into $4 \mathrm{~A}, 4 \mathrm{~B}$, and $4 \mathrm{C}$, which reflect an increasing malignancy potential from low $(2-10 \%)$ to moderate (10-50\%) and high (50-95\%) $(6,7)$. In practice, defining the malignancy of BI-RADS category 4 breast lesions using the available information is essential for an accurate diagnosis and subsequent treatment. In addition to breast mammography, magnetic resonance imaging (MRI) is an essential technique for the diagnosis and subcategory classification of BI-RADS category 4 breast lesions $(8,9)$. However, the accuracy of MRI assessment for this differential diagnosis can be limited by the radiologist's experience.

Radiomics is a new method in medical imaging analysis that can extract and analyze more features from radiographic medical images than the naked eye (10). Existing studies have demonstrated that radiomics (the combination of imaging omics and machine learning methods to construct imaging omics models) can improve the accuracy of clinical diagnoses and provide guidance for clinical decision-making $(11,12)$. $\mathrm{T}_{1} \mathrm{WI}$ and $\mathrm{T}_{2} \mathrm{WI}$ are the basic sequences of MR scanning. T1WI sequence can better display the anatomical structure, and $\mathrm{T}_{2} \mathrm{WI}$ sequence is easy to show the lesions. Diffusionweighted imaging (DWI) is an MRI sequence to reflect the micro movement of water molecules in living tissues, and dynamic contrast enhancement (DCE) can analyze the hemodynamic characteristics of the lesions. Some studies based on DWI and DCE sequences have explored the relationship between breast cancer imaging omics and histopathology $(11,12)$. However, to our best knowledge, previous studies of breast MRI omics mostly focused on the identification of benign and malignant tumors, no report has studied the value of a radiomics-based diagnostic analysis of BI-RADS category 4 breast lesions.

The objective of the present study was to explore the value of breast MRI omics features (e.g., $\mathrm{T}_{1} \mathrm{WI}$ sequence, $\mathrm{T}_{2} \mathrm{WI}$ sequence, DWI sequence, and DCE sequence), histopathology, and clinical manifestations in the assessment of BI-RADS category 4 breast lesions and construct and validate a predictive model.

We present the following article in accordance with the TRIPOD reporting checklist (available at https://dx.doi. org/10.21037/atm-21-5441).

\section{Methods}

The present study was a retrospective cohort study. All procedures performed in this study involving human participants were in accordance with the Declaration of Helsinki (as revised in 2013). The study was approved by the Research Ethics Board of the Second Affiliated Hospital of Dalian Medical University (No. 2020-051). Individual consent for this retrospective analysis was waived.

\section{Patients}

We retrospectively reviewed the medical records of patients treated from May 2017 to December 2019 at the Second Affiliated Hospital of Dalian Medical University. The inclusion criteria and exclusion criteria are shown in Figure 1. In the experiment, the training set and test set contained 71 and 25 cases, respectively.

\section{Image acquisition}

We used 1.5T (GE HDxt) MRI equipment and 3.0T MR scanners (Discovery 750W; GE, USA), with dedicated breast-phased array coils to receive the signals. The patients were in the prone position, undressed, with breasts hanging naturally and placed in the special breast coil and with shoulders relaxed and close to the coil. The scanning sequence and parameters were $T_{1} W I$, fat suppression $T_{2} W I$, DWI, and volume-imaged breast assessment (VIBRANT) dynamic enhanced scans. Patients underwent a $T_{1} W I$ plain scan first, and then a contrast agent (gadodiamide, GdDTPA-BMA, Omniscan, GE Healthcare) was injected through the cubital vein. The contrast agent flow rate was $2 \mathrm{~mL} / \mathrm{s}$ with a dose of $0.2 \mathrm{mmol} / \mathrm{kg}$. The period scan time was $1 \mathrm{~min}$ and $7 \mathrm{~s}$, and the total scan time was $7 \mathrm{~min}$ and $53 \mathrm{~s}$. We selected the most obvious and solid part of the tumor on the DCE image and drew a region of interest (ROI) for the time-intense curve (TIC).

The $1.5 \mathrm{~T}$ scan sequence parameters were as follows: (I) 


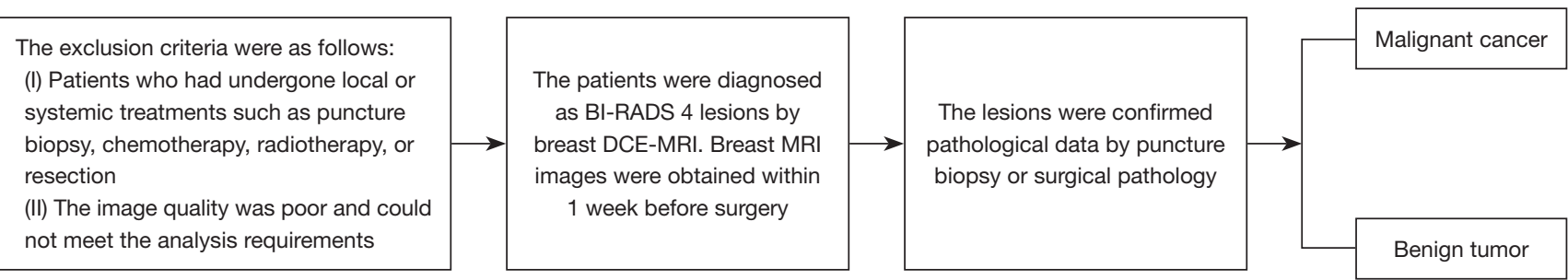

Figure 1 Flow chart of the patient selection process. BI-RADS, Breast Imaging Reporting and Data System; DCE, dynamic contrast enhancement; MRI, magnetic resonance imaging.

conventional plain scan: $\mathrm{T}_{1} \mathrm{WI}, \mathrm{T}_{2} \mathrm{WI}$ sequence; (II) DWI scan parameters: $\mathrm{b}$ value $=800 \mathrm{~s} / \mathrm{mm}^{2}$, repetition time $(\mathrm{TR})$ : 5,600 ms, echo time (TE): $74 \mathrm{~ms}$, matrix: $128 \times 128$, layer thickness: $4.0 \mathrm{~mm}$, interval: $0.4 \mathrm{~mm}$, field of view (FOV): $36 \mathrm{~cm} \times 36 \mathrm{~cm}$, apparent diffusion coefficient (ADC) image acquisition; (III) VIBRANT scanning parameters: TR: $5.0 \mathrm{~ms}$, TE: $2.5 \mathrm{~ms}$, flip angle: $15^{\circ}$, matrix: $288 \times 288$, layer thickness: $1.2 \mathrm{~mm}, \mathrm{FOV}: 32 \mathrm{~cm} \times 32 \mathrm{~cm}$. The $3.0 \mathrm{~T}$ scan parameters were as follows: (I) conventional plain scan: $\mathrm{T}_{1} \mathrm{WI}, \mathrm{T}_{2} \mathrm{WI}$ sequence, (II) DWI scan parameters: $\mathrm{b}$ value $=800 \mathrm{~s} / \mathrm{mm}^{2}$, TR: $5075 \mathrm{~ms}$, TE: $92 \mathrm{~ms}$, matrix: $128 \times 128$, layer thickness: $4.0 \mathrm{~mm}$, interval: $0.4 \mathrm{~mm}, \mathrm{FOV}: 36 \mathrm{~cm} \times$ $36 \mathrm{~cm}$, ADC image acquisition; (III) VIBRANT scanning parameters: TR: $7.6 \mathrm{~ms}$, TE: $3.8 \mathrm{~ms}$, flip angle: $15^{\circ}$, matrix: $288 \times 288$, layer thickness: $1.2 \mathrm{~mm}$, FOV: $32 \mathrm{~cm} \times 32 \mathrm{~cm}$.

\section{Data collection}

\section{Basic characteristics}

The demographic and clinical indicators necessary for clinical decision-making were collected. They included the following 17 items: age, family history, Her-2 expression, pathological type, lymph node metastasis, estrogen receptor (ER) or progesterone receptor (PR) expression, Ki-67 labeling index expression, birth history, lesion diameter $>1 \mathrm{~cm}$, lesion boundaries, location, lesion number $>1$, lesion calcification morphology and distribution, TIC types (inflow, platform, or outflow), menopausal status, and age of menarche. A total of 96 lesions in 92 patients were analyzed, of which 72 were $1.5 \mathrm{~T}$ MR lesions, and 24 were 3.0 $\mathrm{T}$ lesions.

\section{Subtype classification of breast MRI BI-RADS 4 lesions}

The relevant MR images of the patients were analyzed by two diagnostic radiologists who have been engaged in breast imaging diagnosis for more than 6 years. They jointly subtyped the DCE-MRI images while blinded to the pathological results, classifying the breast MRI BIRADS 4 lesions as 4A, 4B, or 4C. The classification criteria were based on general recommendations delivered by the guidance chapter of the mammography section in the BIRADS (6) and the classification method for breast MRI category 4 lesion subtypes (13).

\section{Image omics analysis model}

A flowchart of the image omics algorithm is shown in Figure 2. First, the ROIs on the $\mathrm{T}_{1} \mathrm{WI}, \mathrm{T}_{2} \mathrm{WI}, \mathrm{DWI}$, and DCE-MRI images were delineated. Because breast tumors are irregular and diverse in shape, it is difficult to delineate them accurately and automatically. In practice, it is easier for the reading radiologist to localize the tumor on the MR images. Two radiologists who had been engaged in breast imaging diagnosis for more than 6 years and who were blinded to the pathological results conducted the analyses. They delineated ROIs on all the tumor areas on the MR $\mathrm{T}_{1} \mathrm{WI}, \mathrm{T}_{2} \mathrm{WI}$, DWI, and DCE images and reached final agreements on the delineations. The DCE images were selected by the third-phase enhanced scan after the injection of the contrast agent when the lesion area had generally reached the enhancement peak or the enhancement was more evident, and when the contrast between the lesion and the surrounding normal breast parenchyma was optimal (14) (Figure 3). Then, the breast imaging examination data, including the conventional $\mathrm{T}_{1} \mathrm{WI}, \mathrm{T}_{2} \mathrm{WI}$ sequence, DWI, and DCE MRI were collected and organized.

Second, as suggested by the Image Biomarker Standardization Initiative (IBSI), eight types of histological features were extracted from the ROI of each tumor lesion image, including first-order statistical features, two-dimensional shape features, three-dimensional shape features, gray-level co-occurrence matrix features (GLCM), gray-level run-length matrix (GLRLM), gray-level size zone matrix (GLSZM), gray-level dependence matrix features (GLDM), and Neighboring Gray Tone Difference Matrix 


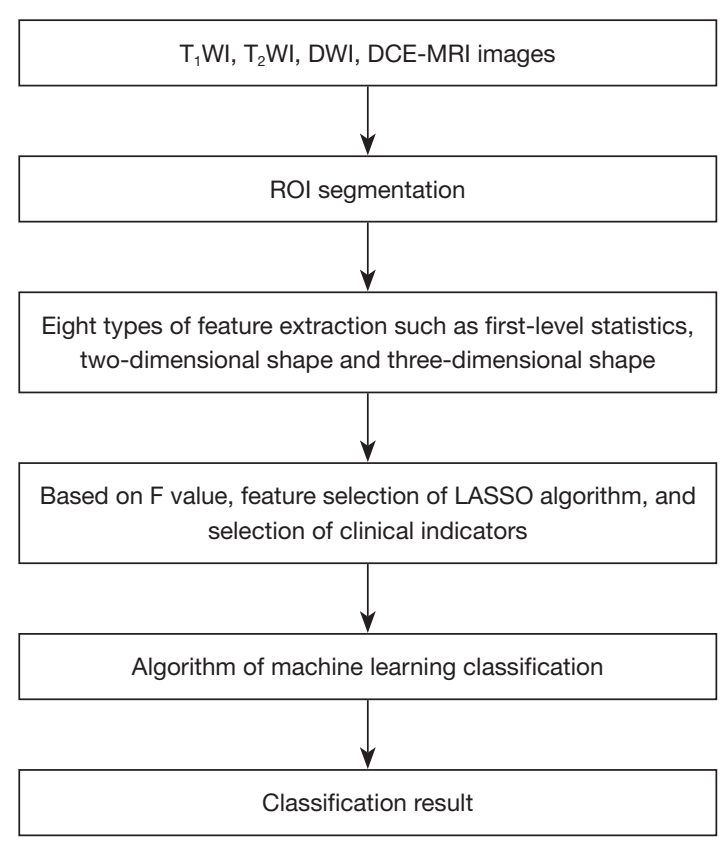

Figure 2 Image omics analysis flow chart. $T_{1} W I, T_{1}$-weighted images; $\mathrm{T}_{2} \mathrm{WI}, \mathrm{T}_{2}$-weighted images; DWI, diffusion-weighted imaging; DCE, dynamic contrast enhancement; MRI, magnetic resonance imaging; ROI, region of interest; LASSO, Least absolute shrinkage and selection operator.

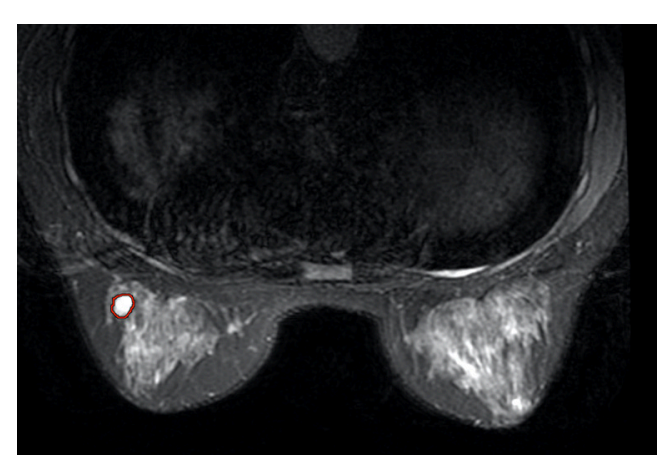

Figure 3 Example of a manually delineated and labeled breast ROI in $\mathrm{T}_{2} \mathrm{WI}$ sequence (red circle). ROI, region of interest; $\mathrm{T}_{2} \mathrm{WI}, \mathrm{T}_{2^{-}}$ weighted images.

(NGTDM) features. The above features can be divided into four categories: first-order features that consider only the intensity of gray values in a ROI, second-order features that consider the pixel-pixel relationship, higher-order features that consider the relationship among the pixels and the environment, and shape features that describe the contours of a ROI. The original image was filtered before feature extraction. The Laplacian of Gaussian, Wavelet, Square, Square root, Logarithm, and Exponential filters were used to perform the filtering. To splice the multi-sequence features, we set a multi-sequence annotation mode. The ROI of a lesion at an anatomical location on $\mathrm{T}_{1} \mathrm{WI}, \mathrm{T}_{2} \mathrm{WI}$, DWI, and DCE MRI sequences is shown in Figure 4.

The next step of the image omics analysis included removing redundant features and selecting the discriminative features using a LASSO algorithm. To facilitate the machine learning modeling, we first normalized all features to the interval $[0,1]$ according to Eq. [1] as follows:

$$
x_{i}^{\prime}=\frac{x_{i}-\min (x)}{\max (x)-\min (x)}
$$

All extracted features were further screened to remove redundant features and obtain the most discriminating features, with the following screening method:

To measure within-group and between-group variance, we computed the sample variance $\mathrm{F}$-value for each feature. If the between-group variance of a feature was significantly greater than the within-group variance, the feature was considered significant. The 200 features with the highest $\mathrm{F}$-values were then selected.

The 200 selected features were used as the input to the LASSO algorithm for further feature filtering. The LASSO algorithm is shown in Eq. [2]:

$$
L_{\alpha}=L(y, h(w x))+\alpha\|w\|_{1}
$$

where $\mathrm{L}$ is the loss of the logistic regression, $\mathrm{w}$ is the weight of the features, $y$ is the predicted value ( $y=0$ for benign and $y=1$ for malignant), and $\alpha$ is the penalty coefficient. The optimal value was obtained by the 5 -fold cross-validation method.

Finally, a machine learning model was constructed to complete the classification. The omics classification model that we built was mainly based on the Darwin Intelligent Research Platform, as shown in Figure 5. The classification model contained artificial intelligence modeling components and clustering components. Moreover, the classification model laid with the support vector machine, which used a linear of the kernel and $\mathrm{c}=1$ (where $\mathrm{c}=$ the penalty coefficient of the error term). We tuned the parameters to obtain the processes and combinations that performed optimally.

\section{Primary outcome}

The primary outcome was the pathological diagnosis of the MRI BI-RADS category 4 breast lesion, which was taken as 

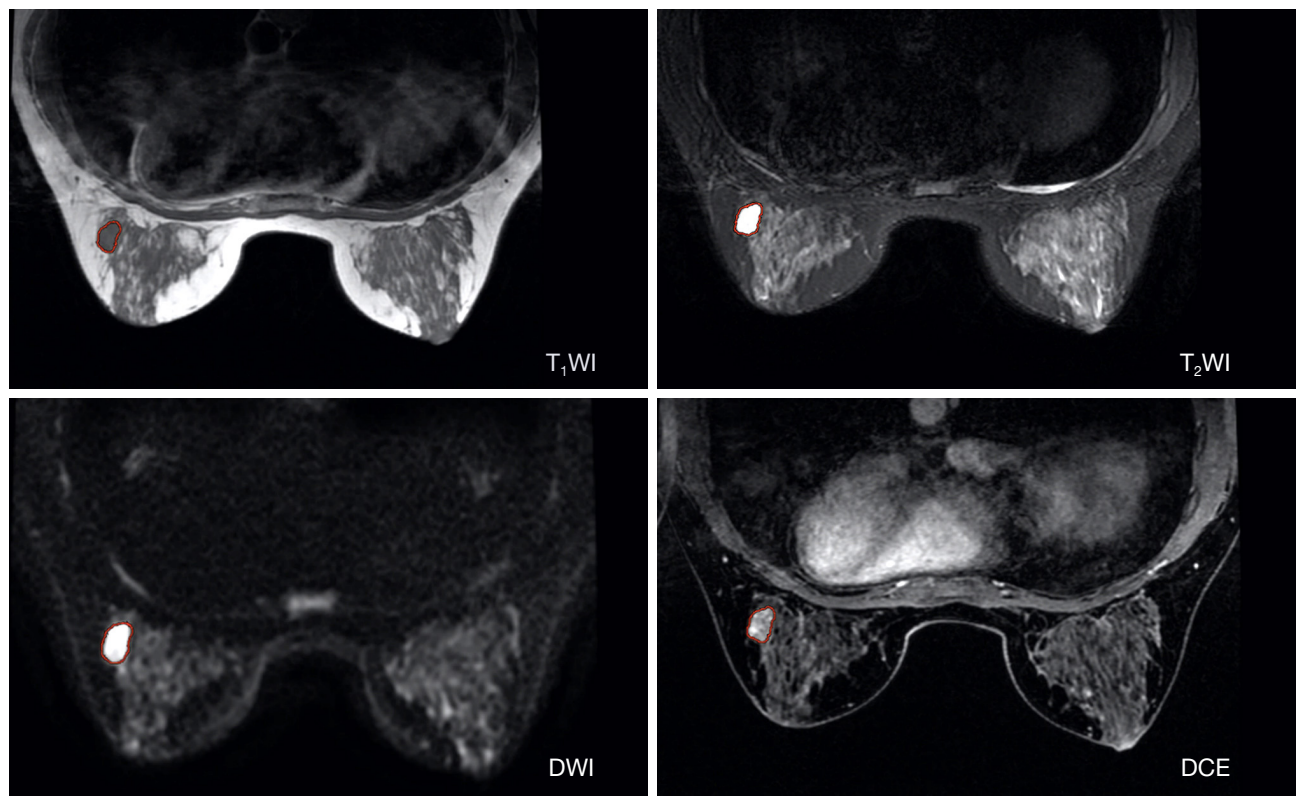

Figure 4 ROI of a lesion at the same anatomical location on $\mathrm{T}_{1} \mathrm{WI}, \mathrm{T}_{2} \mathrm{WI}$, DWI, and DCE MRI sequences (red circle). ROI, region of interest; $\mathrm{T}_{1} \mathrm{WI}, \mathrm{T}_{1}$-weighted images; $\mathrm{T}_{2} \mathrm{WI}, \mathrm{T}_{2}$-weighted images; DWI, diffusion-weighted imaging; DCE, dynamic contrast-enhanced; MRI, magnetic resonance imaging.
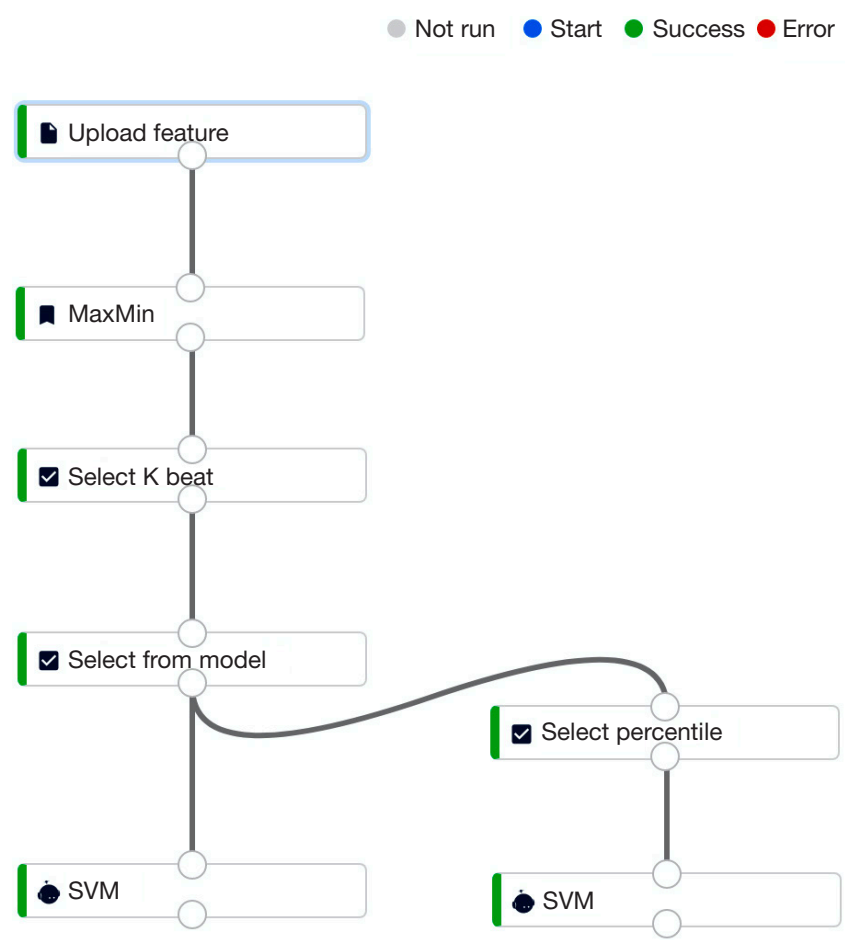

Figure 5 The interface of the Darwin Intelligent Research Platform. The experiment desktop can be used to build rich topological diagrams. SVM, support vector machine. the gold standard. Senior pathologists with at least 5 years of experience and blinded to the MRI results performed the final pathological diagnosis.

\section{Statistical analysis}

We calculated the positive predictive values (PPVs) for the different subtypes of the BI-RADS category 4 lesions and used receiver operating characteristic (ROC) curve analysis to evaluate the effectiveness of the subtype classification methods for distinguishing benign and malignant breast lesions. The PPV was calculated for each subcategory of BIRADS 4 lesions by dividing the true breast cancer diagnosis by the number of subcategories to check their accuracy.

To verify the effectiveness of the features and models selected in this study, we used the following indicators as evaluation criteria: (I) ROC curve of the training set and test set; (II) AUC; (III) P value, and the 95\% confidence interval; (IV) calibration curve, K-fold cross-validation of $\mathrm{k}$ ROC curves, and the $95 \%$ confidence interval. We tested the operator sensitivity and specificity based on the ROC curve. If the ROC curve for the physician was located below the model ROC curve, this meant that the model performed better than the physician; otherwise, the physician's 
Table 1 Classification and PPV of MRI BI-RADS category 4 subtypes among 96 breast lesions

\begin{tabular}{lcccc}
\hline BI-RADS 4 subtype & Total, $\mathrm{n}$ & Benign, $\mathrm{n}$ & Malignant, $\mathrm{n}$ & PPV, \% (cases) \\
\hline BI-RADS 4A & 28 & 26 & 2 & $7.1(2 / 28)$ \\
BI-RADS 4B & 17 & 10 & 7 & $41.2(7 / 17)$ \\
BI-RADS 4C & 51 & 3 & 48 & $94.1(48 / 51)$ \\
\hline
\end{tabular}

PPV, positive predictive value; MRI, magnetic resonance imaging; BI-RADS, Breast Imaging Reporting and Data System.

assessment was superior. Moreover, to illustrate the significance of the results, we used k-fold cross-validation to draw the $95 \%$ confidence interval of the ROC curve. If the interval fell outside the ROC curve, the difference was significant; otherwise, the difference was non-significant.

In addition, we analyzed 17 identified clinical indicators. After normalization and analysis with the Chi-square test, we ranked the indicators by importance and judged the value of each indicator for the differential diagnosis of malignant and benign lesions.

\section{Results}

\section{BI-RADS 4 subtype classification}

Data were collected for 96 MRI BI-RADS category 4 lesions in 92 patients, of which 72 were $1.5 \mathrm{~T}$ MR lesions, and 24 were $3.0 \mathrm{~T}$ lesions. The age of the patients ranged from 30-83 years old, with a median age of 51 years. Postoperative lesion pathology was benign in 39 cases, including 5 cases of benign phyllodes tumors, 10 cases of fibroadenoma, 14 cases of breast disease, 5 cases of intraductal papilloma, 1 case of ductal endothelial normal hyperplasia, 1 case of ductal endothelial atypical hyperplasia, 1 case of nodular fasciitis, 1 case of granulomatous lobulitis, and 1 case of lymphocytic mastitis. Postoperative lesion pathology was malignant in 57 cases, including 49 cases of non-specific invasive breast cancer, 1 case of infiltrating lobular carcinoma, 3 cases of ductal carcinoma in situ, 1 case of invasive breast cancer with neuroendocrine differentiation, and 3 cases of mucinous adenocarcinoma.

Among the 96 breast lesions, the numbers categorized as $4 \mathrm{~A}, 4 \mathrm{~B}$, and $4 \mathrm{C}$ lesions were $28(29.2 \%), 17(17.7 \%)$, and $51(53.1 \%)$, respectively. According to the final pathological diagnosis, the PPVs of the BI-RADS $4 \mathrm{~A}, 4 \mathrm{~B}$, and $4 \mathrm{C}$ subtypes were $7.1 \%(2 / 28), 41.2 \%$ (7/17), and 94.1\% (48/51), respectively. The AUC value for the differential diagnosis of benign and malignant breast lesions by BI-RAD 4 subtype classification was 0.919 , the diagnostic sensitivity was $84.2 \%$, and the diagnostic specificity was $92.3 \%$ (Table 1).

\section{Results of image omics feature filtering}

The results of the LASSO-based feature filtering are presented in Figure 6. It can be seen that a larger penalty coefficient corresponded to a smaller feature coefficient (Figure 6A). Based on the nature of the first-order norm, the unimportant features were removed. Also, the optimal coefficient $\alpha$ was located at the lowest point of $\mathrm{L}$ (Figure $6 B$ ). Figure $6 C$ shows the dimensionality reduction process (based on the penalty factor $\alpha=0.368$ ), compresses the insignificant feature coefficients to 0 , selects the above features, and sorts them according to the size of the coefficients to establish the model.

The absolute value of the Pearson correlation coefficient among the 14 features selected by the LASSO algorithm is shown in Figure $7 A$, and the correlation coefficients among the seven features chosen by the LASSO joint iteration features are shown in Figure 7B. Among them, black represents no correlation, and a light color represents a greater correlation. The correlation between these seven features was small, which validated that we minimized the redundancy of the features. Moreover, due to the absolute value, both positive and negative correlations were observed.

To demonstrate the model's performance, we plotted ROC curves for the training and testing sets of the two methods (Figure 8). The performance of the model processed by LASSO and the wrapped iterative feature selection algorithm was not significantly worse than the performance of the model that took the 14 features as input. However, the other seven features cannot be excluded from reflecting independent predictability in the case of larger data volume, and thus, we mainly used the LASSO algorithm to filter out the 14 features in the subsequent studies.

Among these 14 features (Figure 9), the DWI sequence had only one feature. When we deleted this feature, the performance of the model remained unchanged. The training set yielded an AUC $=0.92$ (95\% CI: 0.84-1), 

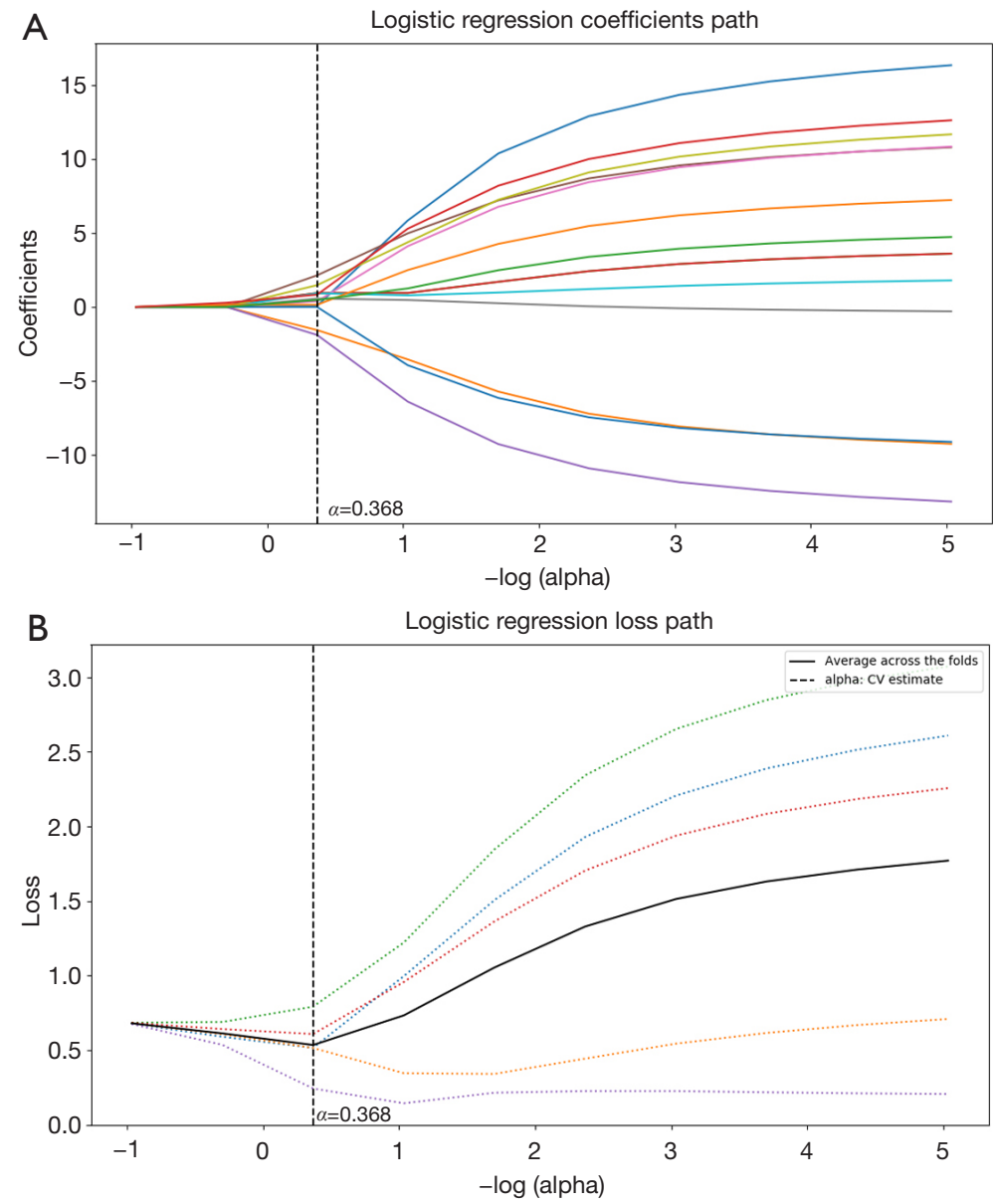

C

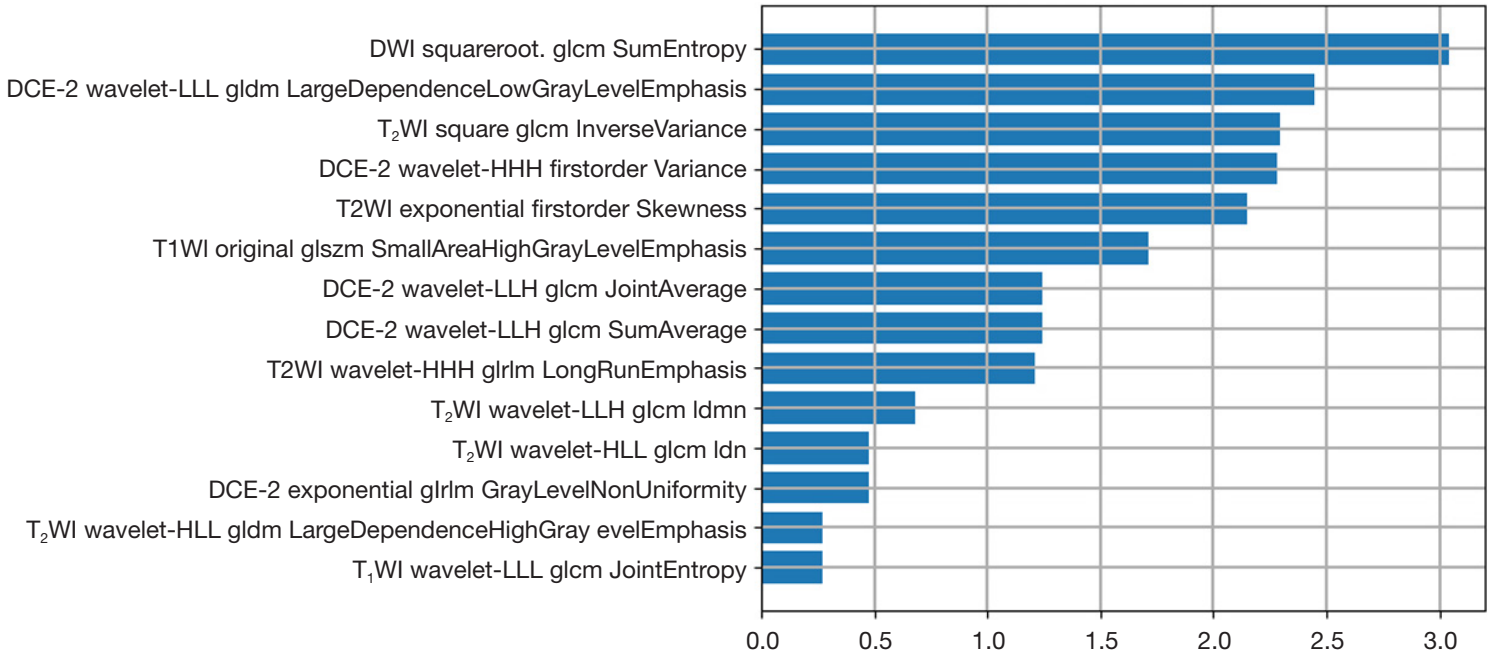

Figure 6 Process of LASSO algorithm-based feature filtering. (A) Plot of the feature coefficient w with increasing penalty factor $\alpha$; (B) selection process that obtains the optimal $\alpha$ by 5 -fold cross-validation, with the optimal coefficient locating at the lowest point of $\mathrm{L}$; (C) the ranking of the selected omics features coefficient $w$ at the optimal coefficient $\alpha=0.368$. DWI, diffusion-weighted imaging; $T_{1} W_{1}$, $T_{1}{ }^{-}$ weighted images; $\mathrm{T}_{2}$ WI, $\mathrm{T}_{2}$-weighted images; DCE, dynamic contrast-enhanced; LASSO, Least absolute shrinkage and selection operator. 


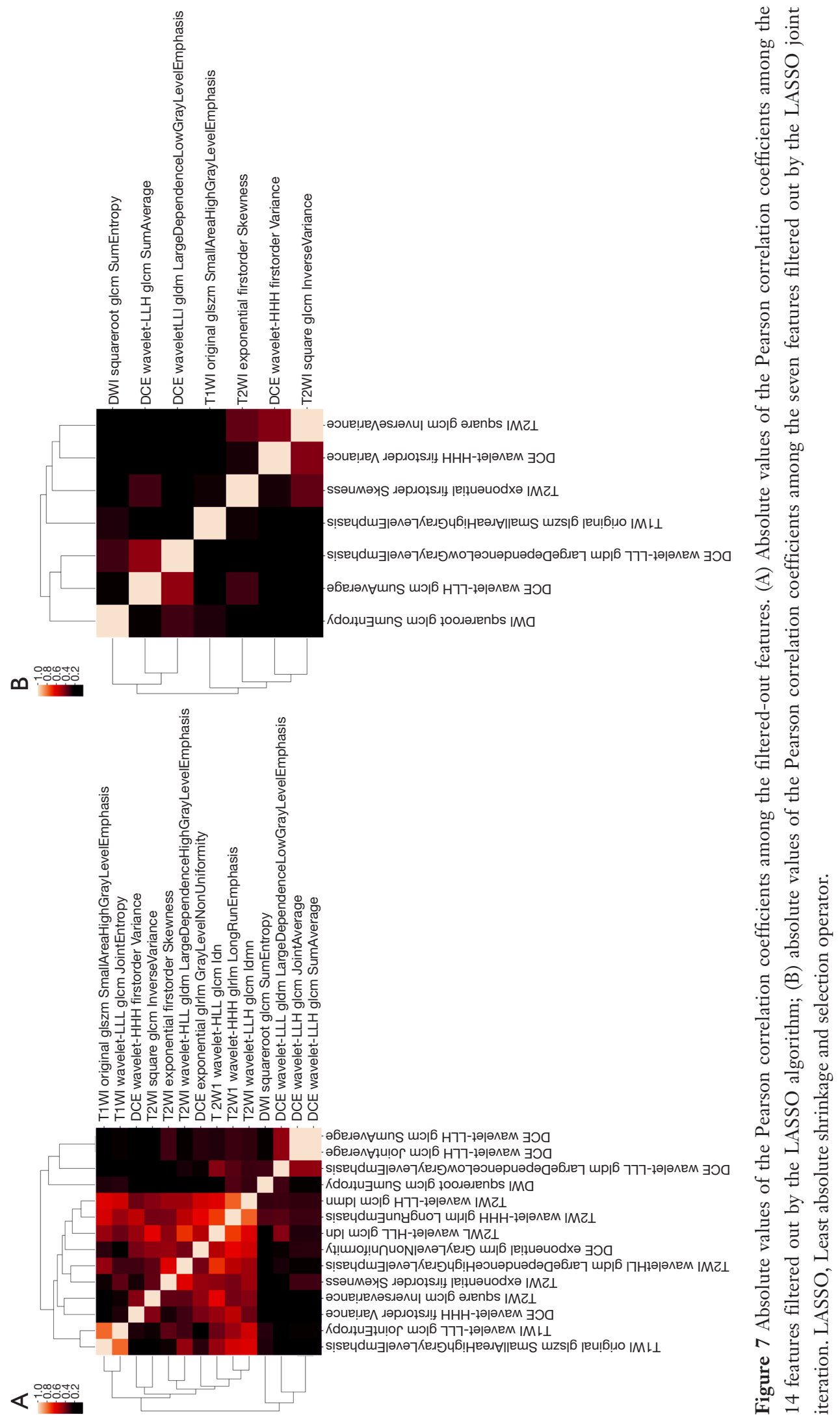

(c) Annals of Translational Medicine. All rights reserved. 


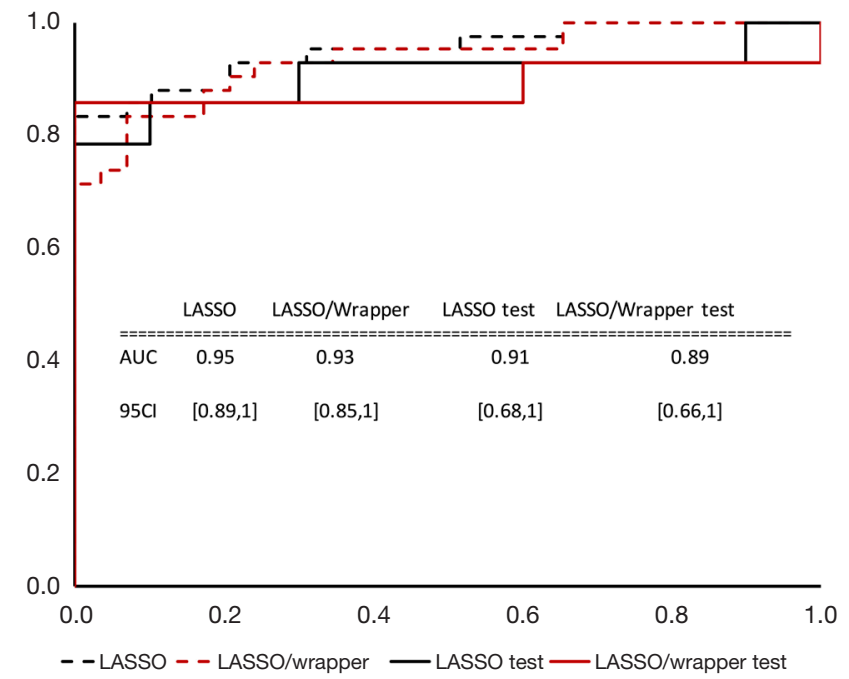

Figure 8 AUC for the model under the two feature-filtering methods. The table in the figure lists the performance of the two sets under the two filtering algorithms. AUC, area under the curve.

and the test set yielded an AUC $=0.91$ (95\% CI: 0.71-1). Therefore, we could save at least one sequence in clinical applications. In addition, we tried the combination of conventional $\mathrm{T}_{1} \mathrm{WI}$ and $\mathrm{T}_{2} \mathrm{WI}$ sequences. The training set yielded an AUC $=0.86$ (95\% CI: 0.74-0.98), and the test set yielded an AUC $=0.90$ (95\% CI: 0.72-1). The model still maintained a very high level with the two sequences. In practice, as $T_{1} W I$ and DCE sequences are commonly combined for increased diagnostic accuracy, we carried out experiments on this combination, which performed best among all the dual-sequence combinations $(\mathrm{AUC}=0.92$, 95\% CI, $0.84-1.0$ in the training set and $\mathrm{AUC}=0.91$, $95 \%$ CI, 0.71-1.0 in the test set). The detailed results are described in Table 2. Although the $\mathrm{T}_{1} \mathrm{WI}$ sequence performed well for the test set, it performed poorly for the training set, and the confidence interval was relatively wide. Therefore, it can be concluded that a certain amount of the deviation was caused by insufficient data.

After the LASSO feature screening process for the 17 identified clinical indicators (Figures 10-12), we found only two indicators, PR and Ki-67 expression, that achieved an AUC almost equal to 1.0. Figure 13 shows the pathological picture of PR and Ki-67 expression in a case of non-specific invasive breast cancer.

\section{Discussion}

The $T_{1} W I, T_{2} W I, D W I$, and DCE sequences on MRI have been widely used to diagnose and analyze breast cancer. It has been suggested that the $T_{2} W I$ signal may reflect the aggressive biological behavior of the tumor (15). DWI is a noninvasive method that can provide information about the diffusion of water molecules in tissues, and the ADC value is a commonly used quantitative parameter of DWI, reflecting the Brownian motion of water molecules in the tissue (15). DCE-MRI can provide information about diseased blood vessels, high-resolution morphological details, and their enhancement characteristics (16). The DCE-MRI sequence has multiple time-phase images after the contrast agent is injected. Different research reports have employed different time phases when performing lesion segmentation and feature extraction, and there is no unified standard. In the present study, we selected the thirdphase enhanced images after the injection of the contrast agent, which can reflect the enhancement characteristics in all seven phases after the injection of the contrast agent (17). $\mathrm{Li}$ et al. found that the DCE-MRI value of malignant breast tumors was higher than that of benign breast tumors (18). Moreover, in another study, it was reported that DCEMRI was able to distinguish high-grade breast cancer from low-grade breast cancer (19). Other studies have shown that when DCE-MRI is combined with the ADC values, the specificity for BI-RADS category 3 and 4 breast lesions diagnosis can be improved (20). In the present study, we obtained a very high diagnostic efficiency by using $T_{1} \mathrm{WI}+$ DCE. Therefore, in practice, we can shorten the patient's examination time and improve the diagnostic accuracy by using $\mathrm{T}_{1} \mathrm{WI}+\mathrm{DCE}$ MRI.

The MRI BI-RADS has not yet refined the classification of the $4 \mathrm{~A}, 4 \mathrm{~B}$, and $4 \mathrm{C}$ subtypes. It has been reported that, according to the guidance chapter of the mammography section in the BI-RADS (13), using the breast DCE-MRI features to refine the classification of BI-RADS category 4 subtypes can improve the diagnosis and differential diagnosis of breast lesions $(13,21)$. We referred to the classification method in the literature (13) and tried to classify the 96 BI-RADS category 4 lesions into subtypes. The results showed that the PPV increased with the increasing subtypes of breast BI-RADS category 4 lesions. This is consistent with the conclusions of other scholars (13).

The imaging representations of different types of breast cancer identified by mammography, ultrasound, and MRI and their relationships with the molecular markers of breast tumors (e.g., ER, PR, and Ki-67) have recently become a global focus of research $(22,23)$. According to the receptor status, breast cancer can be 


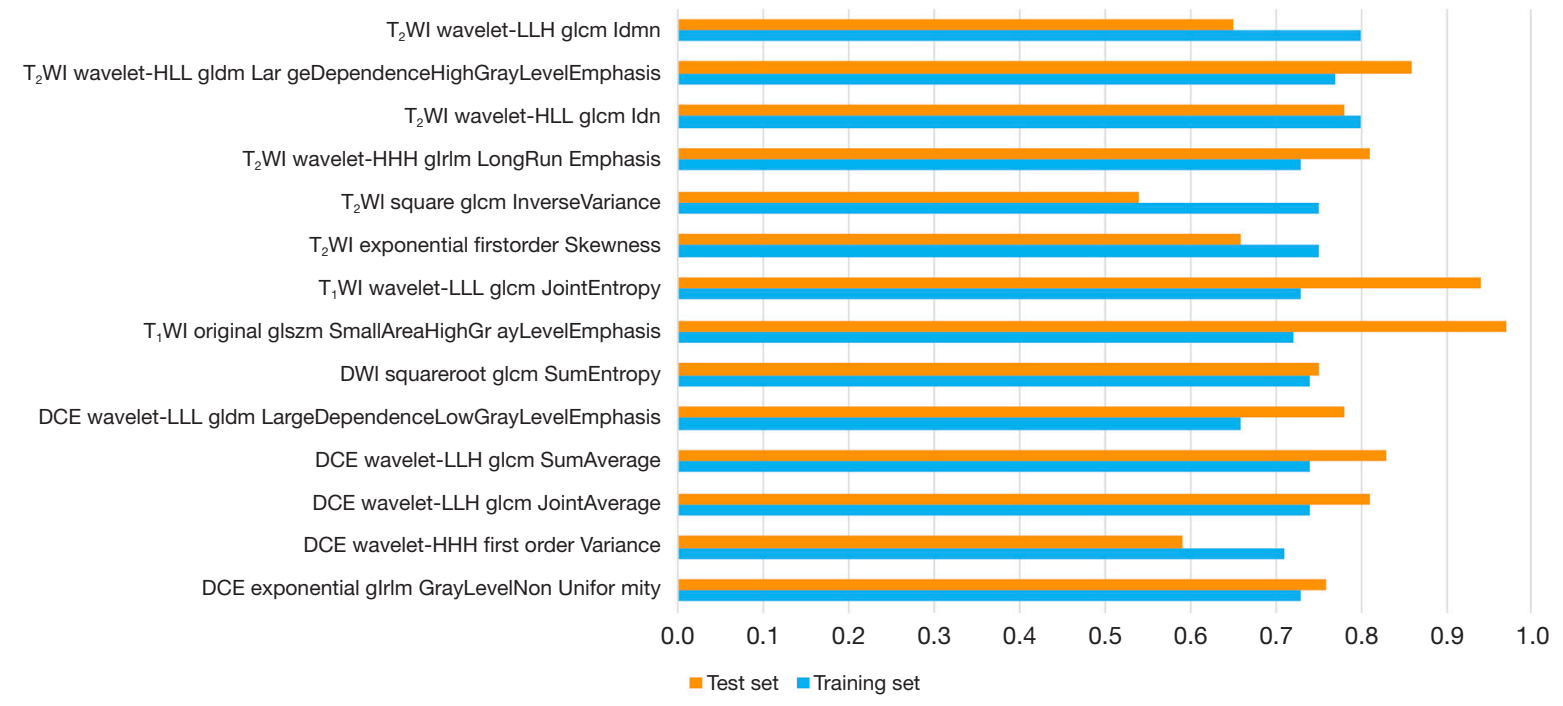

Figure 9 Univariate AUC values of the 14 features chosen by the LASSO algorithm. Blue is the training set, and orange is the test set. $\mathrm{T}_{1} \mathrm{WI}, \mathrm{T}_{1}$-weighted images; $\mathrm{T}_{2} \mathrm{WI}, \mathrm{T}_{2}$-weighted images; DWI, diffusion-weighted imaging; DCE, dynamic contrast-enhanced; LLH, low low high; HLL, high low low; LLL, low low low; HHH, high high high; AUC, area under the curve.

Table 2 Model performance of three commonly used image scanning sequences

\begin{tabular}{lccccc}
\hline & $\mathrm{T}_{1} \mathrm{WI}$ & $\mathrm{T}_{2} \mathrm{WI}$ & $\mathrm{T}_{1} \mathrm{WI}+\mathrm{T}_{2} \mathrm{WI}$ & $\mathrm{T}_{1} \mathrm{WI}+\mathrm{DCE}$ & $\mathrm{T}_{1} \mathrm{WI}+\mathrm{T}_{2} \mathrm{WI}+\mathrm{DCE}$ \\
\hline Training set, AUC (95\% Cl) & $0.74(0.6,0.89)$ & $0.85(0.72,0.97)$ & $0.8(0.76,0.98)$ & $0.89(0.78,1)$ & $0.92(0.84,1.0)$ \\
Test set, AUC (95\% Cl) & $0.96(0.81,1.0)$ & $0.78(0.51,1.0)$ & $0.9(0.75,1.0)$ & $0.94(0.78,1.0)$ & $0.91(0.71,1.0)$ \\
\hline
\end{tabular}

$\mathrm{T}_{1} \mathrm{WI}, \mathrm{T}_{1}$-weighted images; $\mathrm{T}_{2} \mathrm{WI}, \mathrm{T}_{2}$-weighted images; DCE, dynamic contrast enhancement; AUC, area under the curve.

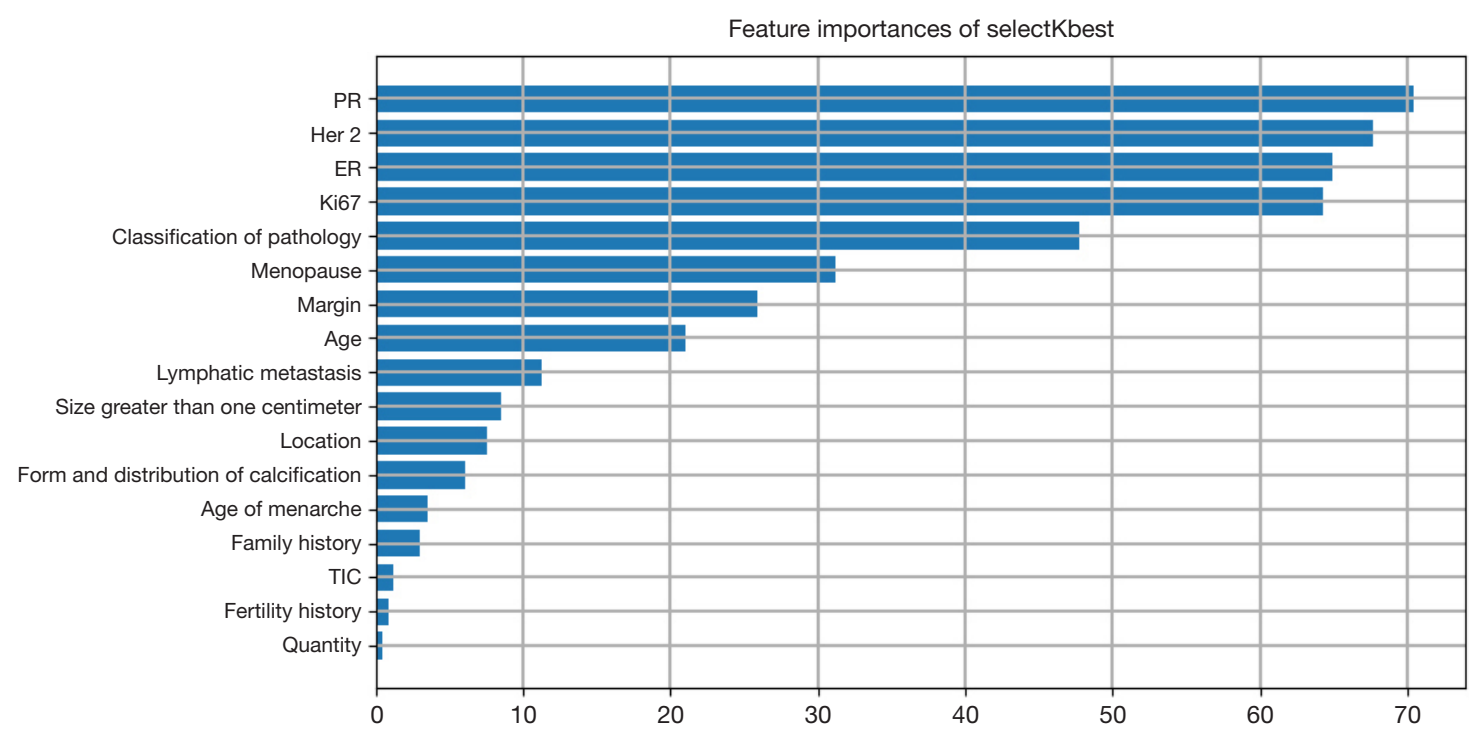

Figure 10 The 17 selected indicators and the gold standard diagnosis of benign and malignant lesions were tested by $\chi^{2}$ test. A larger $\chi^{2}$ value indicates a higher diagnostic value. PR, progesterone receptor; ER, estrogen receptor; TIC, the time-intense curve. 


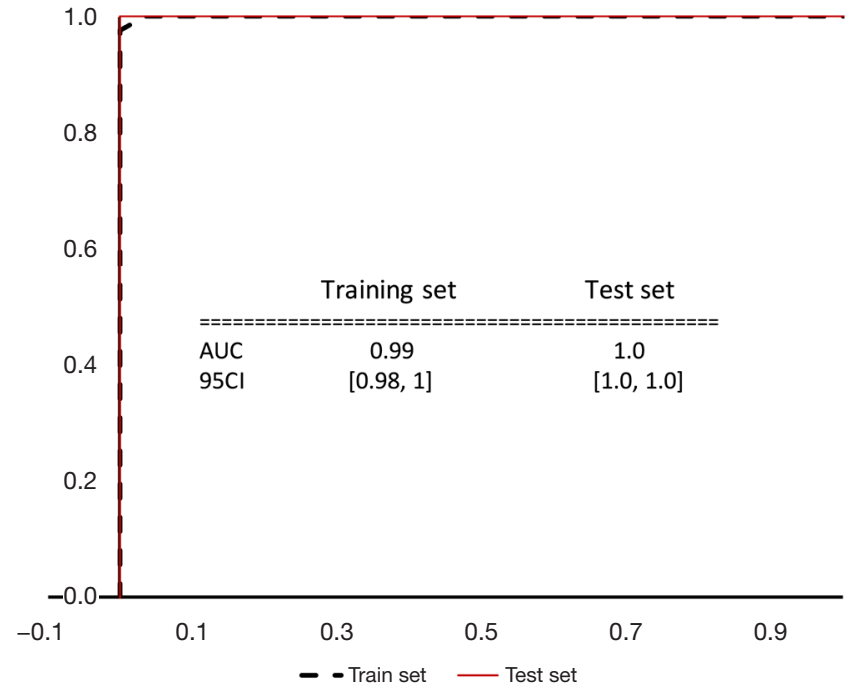

Figure 11 ROC curves for the training set and the test set. ROC, receiver operating characteristic. divided into different molecular subtypes with different biological behaviors and significantly different responses to treatment and prognosis (24). Ki-67 expression provided a guiding role in evaluating the efficacy of neoadjuvant chemotherapy for breast cancer and was closely related to the patients' prognosis (25). Kang et al. conducted a study on 105 patients with invasive breast cancer (26). They showed that PR high-expressing breast cancer had a lower enhancement on MRI, and certain radiological characteristics of DCE-MRI were closely related to the classification of breast cancer. In this study, we found that only two indicators, PR and Ki-67 expression, could achieve an AUC of almost 1.0. The results suggest that the detection of PR and Ki-67 expression plays a significant role in the differential diagnosis of malignant and benign breast lesions when combined with MRI examination.

Imaging examination is an essential part of diagnosing

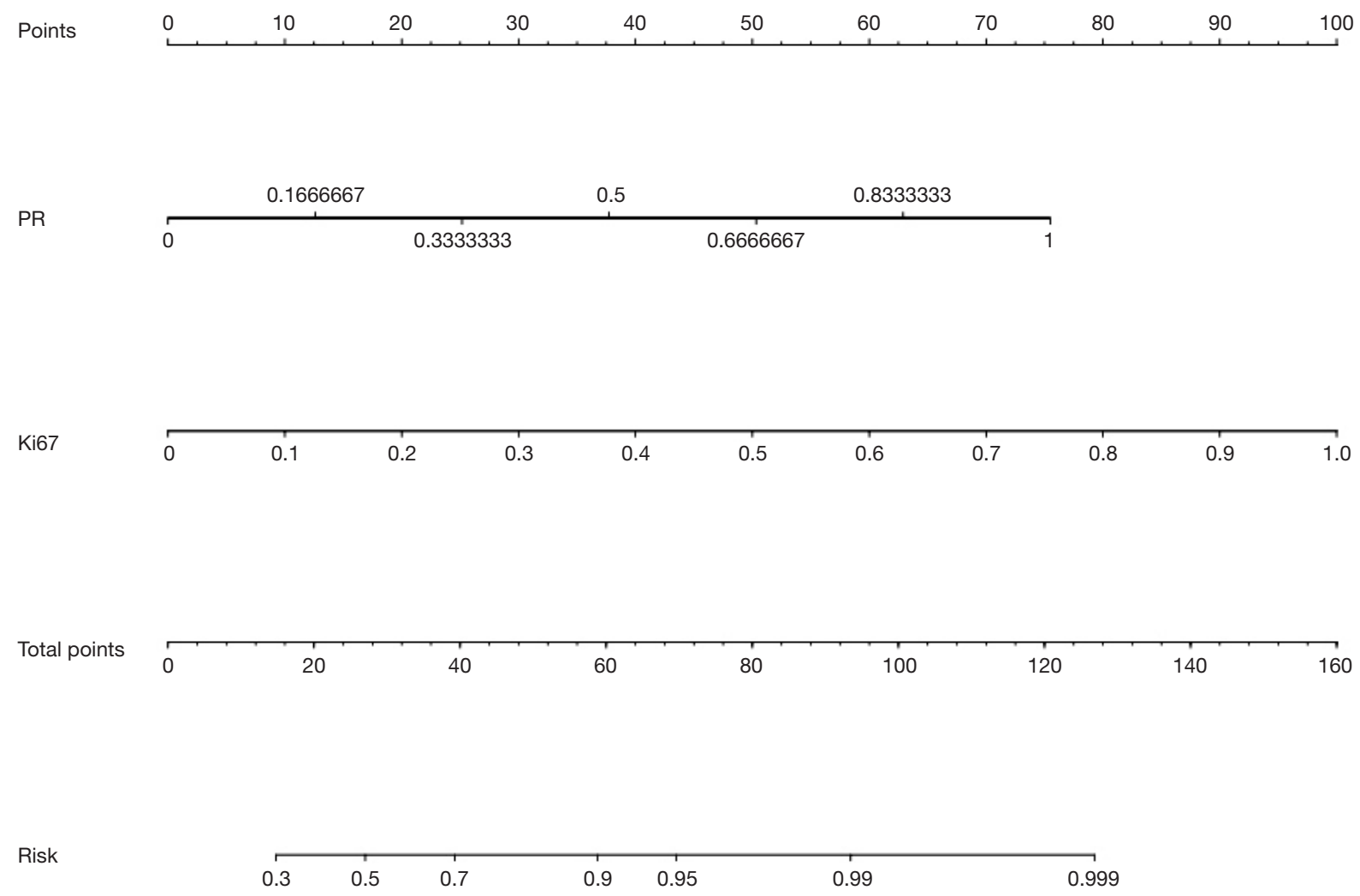

Figure 12 In the nomogram of the model, only PR and Ki-67 expressions are used. PR, progesterone receptor. 


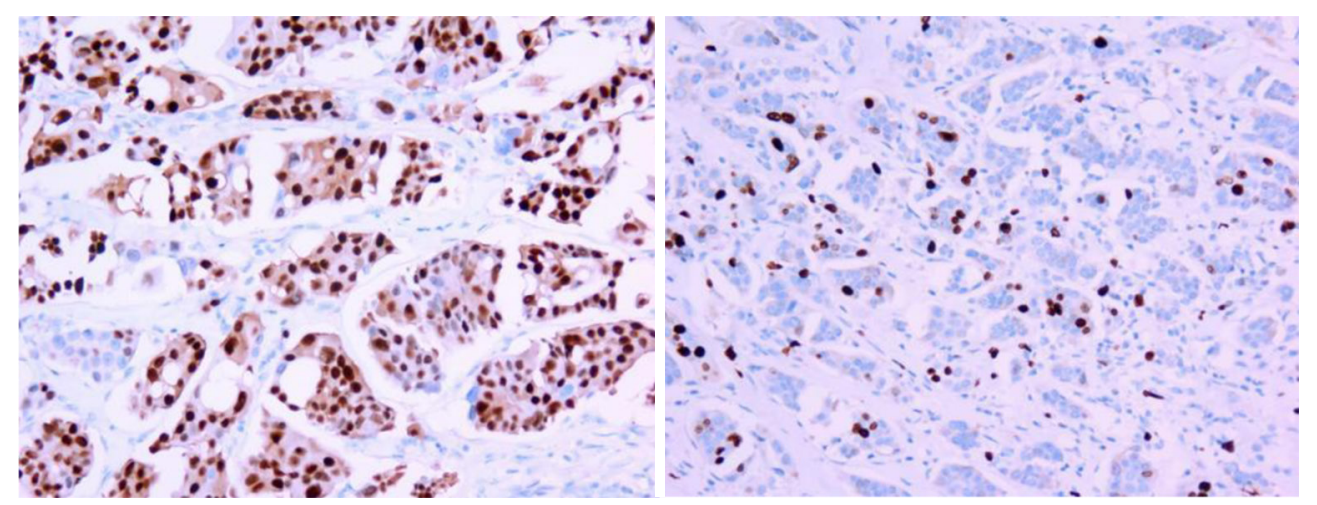

Figure 13 The pathological picture of PR and Ki-67 expression in a case of non-specific invasive breast cancer (PR 95\%, $\times 200 ; \mathrm{Ki} 67$ 10\%, $\times 200)$. Immunochemistry staining. PR, progesterone receptor.

breast cancer and determining prognosis. However, traditional image analysis often relies on the subjective experience of physicians, which can limit diagnostic efficacy. Moreover, much of the deep-level imaging information is not exploited, resulting in a high rate of misdiagnosis (27). Intelligent imaging is based on the medical image and incorporates artificial intelligence, which helps physicians diagnose and treat diseases by segmenting, classifying, quantifying, and characterizing images through machine learning algorithms. Computer-aided diagnosis (CAD) was the initial model that combined medical imaging and artificial intelligence. CAD is currently used in clinical practice for mammography screening, such as detecting micro-calcified lesions (28). In recent years, the field of medical image analysis has grown exponentially with increases in the number of image recognition tools and the size of datasets. These advances have facilitated the development of high-throughput quantitative feature extraction methods and the conversion of images into mineable data, which are subsequently analyzed to provide clinical decision support (29).

Image-omics was proposed by Lambin et al. in 2012 (10) and provides a new approach to image analysis that enables a deep analysis and prediction of image data to aid diagnosis (30). Essentially, this technique extracts quantitative features from medical images at high throughput to characterize tumors and improve diagnostic accuracy. It has been demonstrated that the combination of image-omics and machine learning methods with a lock-constructed imaging omics model can improve the accuracy of diagnosis and guide clinical decision-making $(11,12,31)$. The present study constructed feature and processing, feature screening, and machine learning classification models based on the Darwin Intelligent Research Platform to classify breast BI-RADS category 4 lesions more accurately and reliably. The novelty of this approach lies with the use of the LASSO algorithm to select the most discriminative features and further adopt the iterative feature selection process. Our experimental results demonstrated that representative features could be extracted from a large number of features by the LASSO and wrapped iterative feature selection algorithm without compromising the diagnostic accuracy of the model. Meanwhile, for clinical examination, only PR and Ki-67 detection were needed to achieve an AUC of almost 1.0.

The traditional image analysis of BI-RADS category 4 breast lesions is based on doctors' subjective experience judgment, the diagnostic efficiency is limited, and more valuable image information have not been mined and utilized. Radiomics can extract and analyze more information from radiographic medical images than radiologists, and performs better in disease prognosis. This study has some limitations, as the present study was a retrospective study, confounding variables are difficult to control, and research bias is inevitable. Additionally, the sample size was less than 100 patients, which may have decreased the study power. Prospective studies are needed to control confounding variables, to further evaluate the value of MRI omics features and clinical characteristics in the assessment of BI-RADS category 4 breast lesions. In the future, we intend to collect more data to confirm the findings of this study and attempt to extend the proposed model to other disease classification and diagnostic processes. 


\section{Conclusions}

High diagnostic performance can be achieved for distinguishing malignant BI-RADS type 4 breast lesions using a combination of $T_{1} W I$ and DCE MRI sequences. The addition of PR and Ki-67 detection can improve the diagnostic accuracy of this MRI approach for BI-RADS category 4 breast lesions to near perfect. These findings have important clinical implications for breast cancer diagnosis.

\section{Acknowledgments}

Funding: This work was supported in part by the National Natural Science Foundation of China (61572104), the Natural Science Foundation of Liaoning Province (2019MS045), the Fundamental Research Funds for the Central Universities (DUT21YG106), the Natural Science Foundation of Liaoning Province (2019ZD0933) and the Scientific Research Project of the Educational Department of Liaoning Province (LZ2020025).

\section{Footnote}

Reporting Checklist: The authors have completed the TRIPOD reporting checklist. Available at https://dx.doi. org/10.21037/atm-21-5441

Data Sharing Statement: Available at https://dx.doi. org/10.21037/atm-21-5441

Conflicts of Interest: All authors have completed the ICMJE uniform disclosure form (available at https://dx.doi. org/10.21037/atm-21-5441). The authors have no conflicts of interest to declare.

Ethical Statement: The authors are accountable for all aspects of the work in ensuring that questions related to the accuracy or integrity of any part of the work are appropriately investigated and resolved. All procedures performed in this study involving human participants were in accordance with the Declaration of Helsinki (as revised in 2013). The study was approved by the Research Ethics Board of the Second Affiliated Hospital of Dalian Medical University (No. 2020-051). Individual consent for this retrospective analysis was waived.

Open Access Statement: This is an Open Access article distributed in accordance with the Creative Commons Attribution-NonCommercial-NoDerivs 4.0 International License (CC BY-NC-ND 4.0), which permits the noncommercial replication and distribution of the article with the strict proviso that no changes or edits are made and the original work is properly cited (including links to both the formal publication through the relevant DOI and the license). See: https://creativecommons.org/licenses/by-nc-nd/4.0/.

\section{References}

1. Chen $W$, Zheng R, Baade PD, et al. Cancer statistics in China, 2015. CA Cancer J Clin 2016;66:115-32.

2. Sung H, Ferlay J, Siegel RL, et al. Global Cancer Statistics 2020: GLOBOCAN Estimates of Incidence and Mortality Worldwide for 36 Cancers in 185 Countries. CA Cancer J Clin 2021;71:209-49.

3. Byers T, Wender RC, Jemal A, et al. The American Cancer Society challenge goal to reduce US cancer mortality by 50\% between 1990 and 2015: Results and reflections. CA Cancer J Clin 2016;66:359-69.

4. Wörmann B. Breast cancer: basics, screening, diagnostics and treatment. Med Monatsschr Pharm 2017;40:55-64.

5. Edwards SD, Lipson JA, Ikeda DM, et al. Updates and revisions to the BI-RADS magnetic resonance imaging lexicon. Magn Reson Imaging Clin N Am 2013;21:483-93.

6. D'Orsi C, Bassett L, Feig S. Breast imaging reporting and data system (BI-RADS). Breast Imaging Atlas 2018.

7. Burnside ES, Sickles EA, Bassett LW, et al. The ACR BIRADS experience: learning from history. J Am Coll Radiol 2009;6:851-60.

8. Honda M, Kataoka M, Kawaguchi K, et al. Subcategory classifications of Breast Imaging and Data System (BI-RADS) category 4 lesions on MRI. Jpn J Radiol 2021;39:56-65.

9. Leithner D, Wengert G, Helbich T, et al. MRI in the Assessment of BI-RADS® 4 lesions. Top Magn Reson Imaging 2017;26:191-9.

10. Lambin P, Rios-Velazquez E, Leijenaar R, et al. Radiomics: extracting more information from medical images using advanced feature analysis. Eur J Cancer 2012;48:441-6.

11. De Bernardi E, Buda A, Guerra L, et al. Radiomics of the primary tumour as a tool to improve $18 \mathrm{~F}-\mathrm{FDG}-\mathrm{PET}$ sensitivity in detecting nodal metastases in endometrial cancer. EJNMMI Res 2018;8:86.

12. Ferreira Junior JR, Koenigkam-Santos M, Cipriano FEG, et al. Radiomics-based features for pattern recognition of lung cancer histopathology and metastases. Comput 
Methods Programs Biomed 2018;159:23-30.

13. Maltez de Almeida JR, Gomes AB, Barros TP, et al. Subcategorization of Suspicious Breast Lesions (BI-RADS Category 4) According to MRI Criteria: Role of Dynamic Contrast-Enhanced and Diffusion-Weighted Imaging. AJR Am J Roentgenol 2015;205:222-31.

14. Nie K, Chen JH, Yu HJ, et al. Quantitative analysis of lesion morphology and texture features for diagnostic prediction in breast MRI. Acad Radiol 2008;15:1513-25.

15. Le Bihan D, Breton E, Lallemand D, et al. Separation of diffusion and perfusion in intravoxel incoherent motion MR imaging. Radiology 1988;168:497-505.

16. Macura KJ, Ouwerkerk R, Jacobs MA, et al. Patterns of enhancement on breast MR images: interpretation and imaging pitfalls. Radiographics 2006;26:1719-34; quiz 1719.

17. Fan M, Yuan W, Zhao W, et al. Joint Prediction of Breast Cancer Histological Grade and Ki-67 Expression Level Based on DCE-MRI and DWI Radiomics. IEEE J Biomed Health Inform 2020;24:1632-42.

18. Li H, Sun H, Liu S, et al. Assessing the performance of benign and malignant breast lesion classification with bilateral TIC differentiation and other effective features in DCE-MRI. J Magn Reson Imaging 2019;50:465-73.

19. Shin SU, Cho N, Kim SY, et al. Time-to-enhancement at ultrafast breast DCE-MRI: potential imaging biomarker of tumour aggressiveness. Eur Radiol 2020;30:4058-68.

20. Dijkstra H, Dorrius MD, Wielema M, et al. Quantitative DWI implemented after DCE-MRI yields increased specificity for BI-RADS 3 and 4 breast lesions. J Magn Reson Imaging 2016;44:1642-9.

21. Vanel D. The American College of Radiology (ACR) Breast Imaging and Reporting Data System (BI-RADS): a step towards a universal radiological language? Eur J Radiol 2007;61:183.

22. Goldhirsch A, Wood WC, Coates AS, et al. Strategies

Cite this article as: Cui Q, Sun L, Zhang Y, Zhao Z, Li S, Liu Y, Ge H, Qin D, Zhao Y. Value of breast MRI omics features and clinical characteristics in Breast Imaging Reporting and Data System (BI-RADS) category 4 breast lesions: an analysis of radiomics-based diagnosis. Ann Transl Med 2021;9(22):1677. doi: 10.21037/atm-21-5441 for subtypes--dealing with the diversity of breast cancer: highlights of the St. Gallen International Expert Consensus on the Primary Therapy of Early Breast Cancer 2011. Ann Oncol 2011;22:1736-47.

23. Wu M, Ma J. Association Between Imaging Characteristics and Different Molecular Subtypes of Breast Cancer. Acad Radiol 2017;24:426-34.

24. Anderson WF, Rosenberg PS, Prat A, et al. How many etiological subtypes of breast cancer: two, three, four, or more?. J Natl Cancer Inst 2014;106:dju165.

25. Mu K, Li L, Yang Q, et al. A standardized method for quantifying proliferation by $\mathrm{Ki}-67$ and cyclin A immunohistochemistry in breast cancer. Ann Diagn Pathol 2015;19:243-8.

26. Kang SR, Kim HW, Kim HS. Evaluating the Relationship Between Dynamic Contrast-Enhanced MRI (DCE-MRI) Parameters and Pathological Characteristics in Breast Cancer. J Magn Reson Imaging 2020;52:1360-73.

27. Arnaout A, Catley C, Booth CM, et al. Use of Preoperative Magnetic Resonance Imaging for Breast Cancer: A Canadian Population-Based Study. JAMA Oncol 2015;1:1238-50.

28. Giger ML. Update on the potential of computer-aided diagnosis for breast cancer. Future Oncol 2010;6:1-4.

29. Gillies RJ, Kinahan PE, Hricak H. Radiomics: Images Are More than Pictures, They Are Data. Radiology 2016;278:563-77.

30. Aerts HJ, Velazquez ER, Leijenaar RT, et al. Decoding tumour phenotype by noninvasive imaging using a quantitative radiomics approach. Nat Commun 2014;5:4006.

31. Chen LK. Machine Learning Improves Analysis of MultiOmics Data in Aging Research and Geroscience. Arch Gerontol Geriatr 2021;93:104360.

(English Language Editor: D. Fitzgerald) 